\title{
Simpler, faster, more reliable photosensor circuits
}

\author{
YORK MAKSIK \\ Brown University, Providence, Rhode Island
}

\begin{abstract}
Experimental psychologists have used infrared sensors for many purposes. A new set of readily available components has recently made circuit design and computer interfacing easier, made circuits more reliable, and enabled faster reaction times. Circuits with the new components are compared with equivalent circuits with the old components, and methods of interfacing to the IBM PC and Macintosh are discussed.
\end{abstract}

Infrared emitter-detector pairs have been used in psychology laboratories for many years for many purposes. They have been used, for example, to detect the motion of subjects in activity-monitoring boxes, to count the number of turns of a running wheel, and to determine the position of pigeon pecks on video monitors. They have also been used as bounce-free response keys in a number of experiments.

\section{The Single Pair Tapping-Box Circuit}

Yet, although these devices have been extremely useful traditionally, they have suffered from problems that have limited their applicability. The biggest problem is their sensitivity to background infrared radiation. A typical configuration of an emitter-detector pair used as a beam-break detector in a tapping box is shown in Figure 1. The tapping box is simply a single emitter-detector pair mounted on a hand-held box used to measure rates of human finger tapping. When the infrared beam from the emitter is blocked by the subject's finger, the phototransistor turns off and drives the output to ground. A computer measures the state of the output and acts accordingly. When this circuit is used in incandescent lighting conditions, the background infrared radiation from the incandescent bulb is sometimes sufficient to keep the phototransistor from turning off when the emitter beam is blocked. This results in missed responses.

Several solutions to the background radiation problem have been devised. The simplest is to block most of the external light by shielding the phototransistors with an infrared-opaque material in all but one direction. However, if any significant distance between the emitter and detector is required (e.g., in an activity-monitoring box or a touch frame), it will still be possible for external light to affect the detector. A second solution is to substitute R1, in Figure 1 , with a $100-\mathrm{k} \Omega$ potentiometer and adjust the sensitivity of the detector circuit. For some applications, this is sufficient; if there are changing light conditions, however, or if many beam-break detectors are in use at the

Correspondence should be addressed to York A. Maksik, Department of Psychology, Box 1853, Brown University, Providence, RI 02912. same time, it may not be practical to adjust and test the circuit before each use. A more serious problem is that changing the sensitivity of the circuit also changes its response time. In extremely time-sensitive experiments, such as the human tapping experiments for which this circuit was originally designed, this could mean that 2 subjects who responded at exactly the same time under different lighting conditions would have their responses recorded several milliseconds apart.

Probably the best solution to the background radiation problem is that illustrated in Figure 2. In this circuit diagram, the simple infrared LED has been replaced with a circuit that causes the LED to blink at an adjustable, preset frequency. The simple detector circuit has been replaced with a circuit that acts as a signal amplifier and missing-pulse detector. When both the emitter and the detector are set at the same frequency, the detector ignores any infrared radiation that is not pulsing at its set frequency. When the beam of the emitter is broken for longer than one cycle of its preset frequency, the detector's output goes to ground; otherwise it remains high. This is the solution most often used by industry in commercial products such as VCR remote controls and lightwave transceivers. The circuit of Figure 2 can be used in nearly any lighting situation in which the background infrared radiation and the emitter do not pulse at the same frequency, including outdoors. The pulse frequencies of the emitter and detector are usually set between 20,000 and 60,000 pulses per second. If a very fast circuit is needed, the frequency of both the emitter and the detector can be set as high as 100,000 pulses per second. With the use of lenses, the emitter and detector can be separated by as much as several hundred feet. A problem with this solution remains, however; it requires a comparatively large number of parts and may need frequent calibration, owing to operational amplifier drift. If one is building an application that requires a large number of infrared emitterdetector pairs, the wiring and calibration can easily get out of hand.

Recently, a new set of optoelectronic components has made the design of infrared beam-break circuits and the circuits themselves faster and more reliable, as a result of the incorporation of the solution of Figure 2 in their 

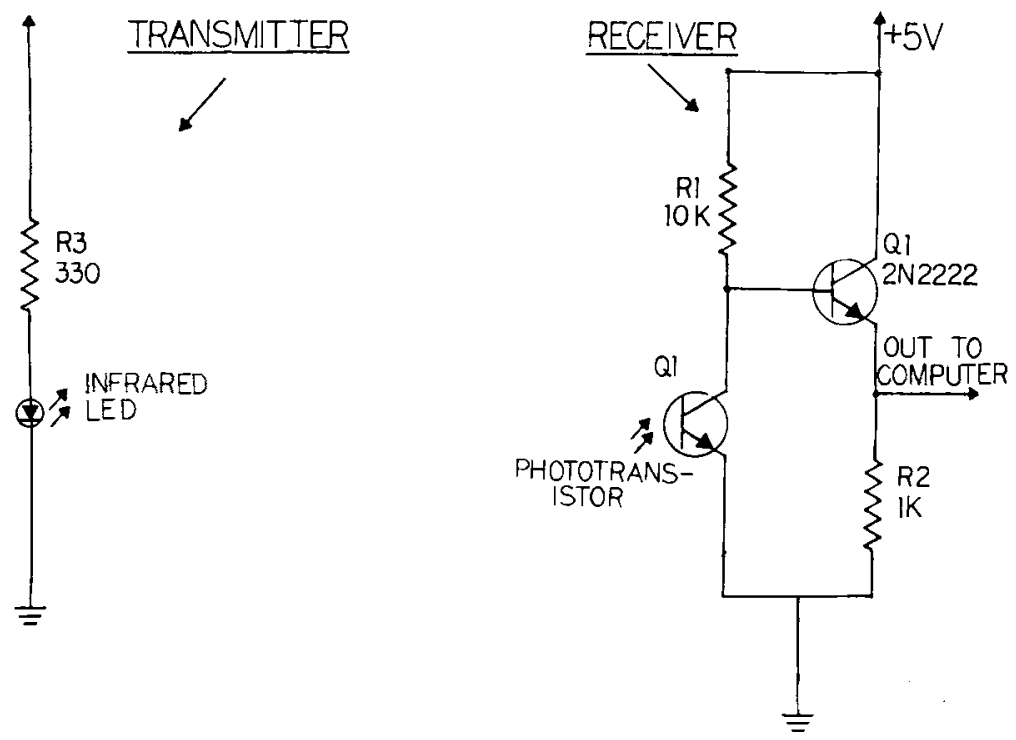

Figure 1. Tapping-box circuit with old components.

\section{TRANSMITTER}
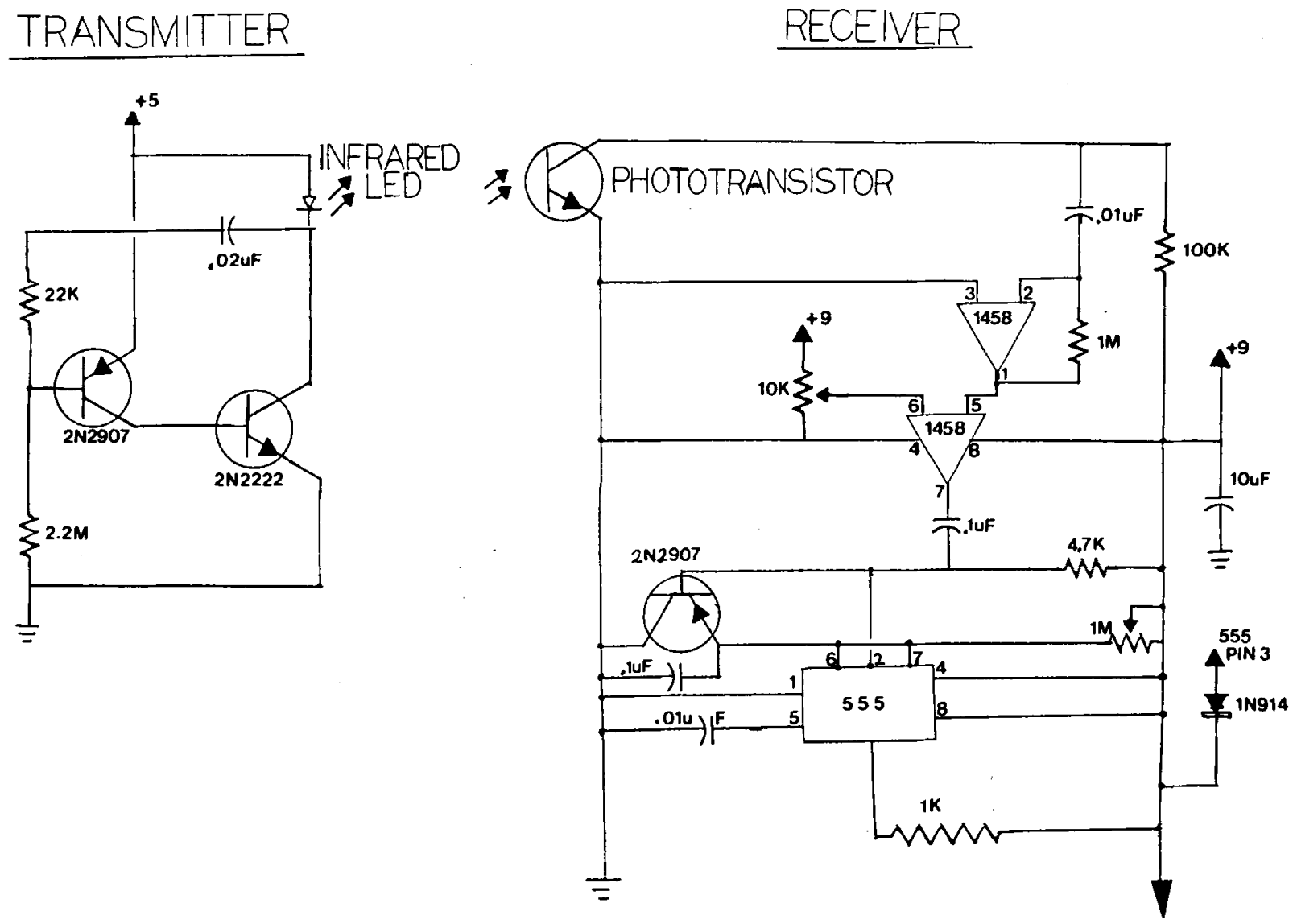

Figure 2. Tapping-box circuit with old components; pulse generator and missing-pulse detector added. 
internal design. The new infrared emitter now contains a pulse generator similar to that in Figure 2, that emits a series of pulses of infrared frequency light rather than emitting just a steady beam of light as does a standard infrared LED. The emitter module is available in 20 different pulse frequencies ranging from 20 to $60 \mathrm{kHz}$ and comes in a package indistinguishable from a standard infrared LED. The infrared detector module now contains a photodiode, a signal amplifier, a limiter, a pulse generator, a demodulator, an integrator, and a comparator all in a package with the same size and shape as that of the ordinary phototransistor in Figure 1. This collection of components behaves much as does the detector circuit of Figure 2. The detector is also available in $\mathbf{2 0}$ different models, each designed to receive one of the specific pulse frequencies of the respective emitter. The new emitter (Radio Shack No. 276-143, Fairchild No. SEP8703-1) can be used in the same ways that an infrared LED can be used, and the new detector unit (Radio Shack No. 276-137, Fairchild GPIU52X, or, without amplifier, Radio Shack No. 276-145) can be used instead of a standard phototransistor. The final version of the tapping circuit with the new components is shown in Figure 3. This circuit diagram is extremely simple, and it is in fact identical to that in Figure 1; however, it has all of the functionality and background radiation immunity of the circuit in Figure 2.

\section{Testing the New Tapping-Box Circuit}

Figure 4 shows a comparative test of two tapping-box circuits, the circuit in Figure 1 with the older components and the circuit of Figure 3 with the newer components. The test procedure was as follows. The emitter side of each circuit was connected through a solid-state relay under computer control. The output of each circuit was then hooked to a digital input port connected to the same computer in order to measure when the detector of each circuit could no longer detect the presence of the emitter beam. Both circuits were placed 5 in. under a $100-W$ incandescent bulb, and the computer cut power to the emitter for 2 out of every $500 \mathrm{msec}$. This was repeated 3,000 times, and the results are summarized in Figure 4. The circuit built with the old components missed nearly 1 out of every 30 simulated beam breaks, whereas the circuit built with the new components missed slightly more than 1 out of 1,000 . When the circuit in Figure 2 was tested in the same way, it missed 5 times as many as did the circuit in Figure 3, or 20 out of 3,000. When the off time was increased from $2 \mathrm{msec}$ to a slightly more realistic $4 \mathrm{msec}$, the new circuit never misread while the others continued to misread.

\section{Designing Multiple-Beam Applications with the New Components}

Although the problem of background radiation has been solved, the problem of emitter beam crossover still exists for applications that involve several emitter-detector pairs. A good example of how this crossover problem occurs is shown in Figure 5; it shows the $x$-axis of a device called a touch frame.

A touch frame is an array of closely spaced infrared emitter-detector pairs arranged around the outside edge of a video monitor. Any touch to a stimulus appearing on the video monitor will interrupt one or more of the beams in both the horizontal and the vertical directions; this gives a cartesian mapping of where the response occurred in relation to where the stimulus was presented. As can be seen in Figure 5, when several emitters are placed next to each other and face several detectors also placed next to each other, each detector can receive light

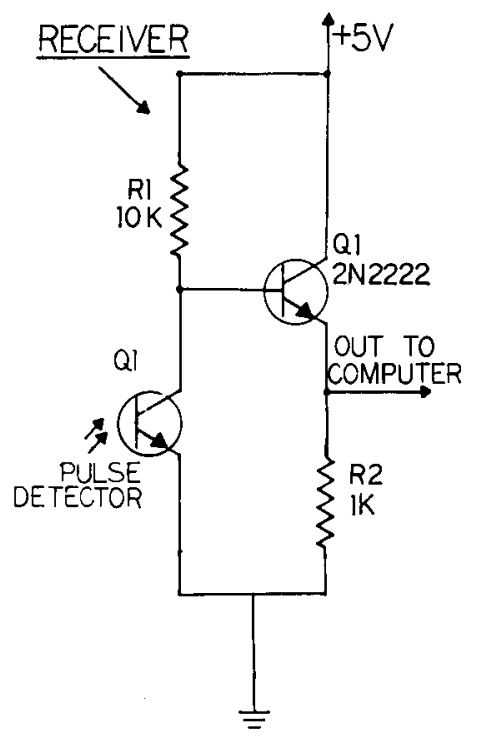

Figure 3. Tapping-box circuit with new components. 


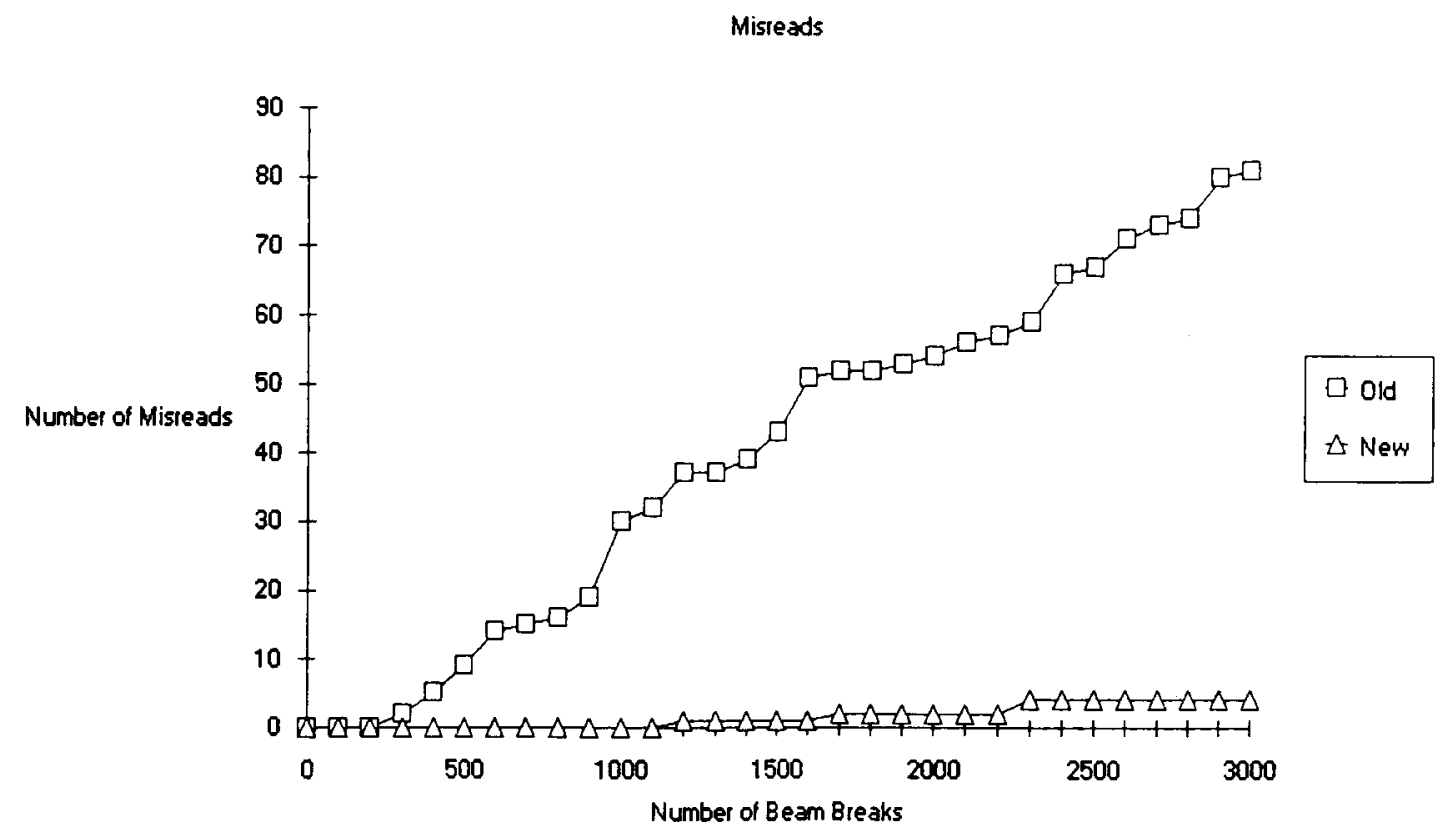

Figure 4. The number of errors in reading beam breaks with old and new components. See text for details.

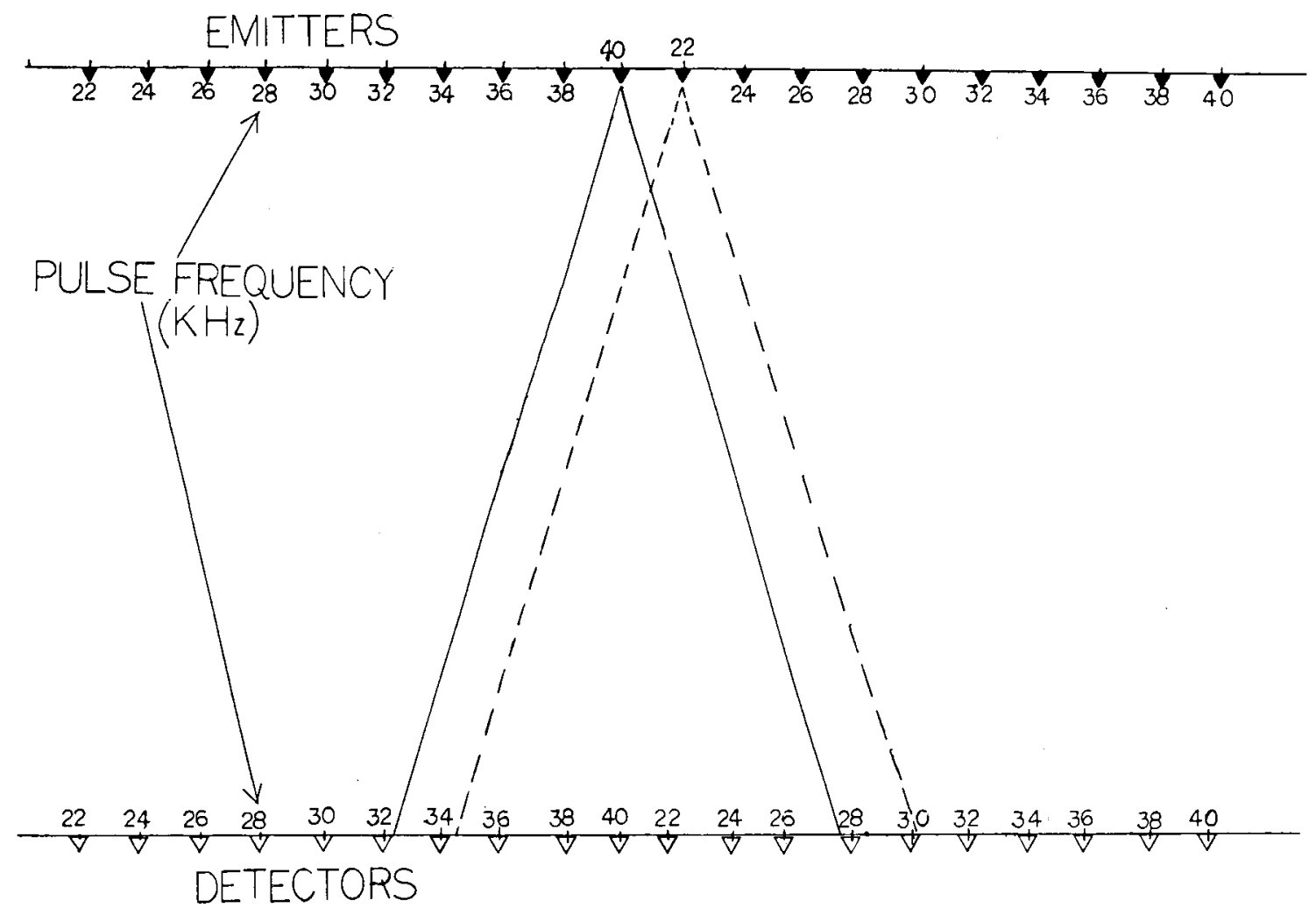

Figure 5. $x$-axis of a touch frame, showing how emitter beam crossover occurs, and an arrangement of emitters and detectors to combat the problem. 
from several emitters. This means that if an object blocks one emitter, its respective detector may continue to receive sufficient radiation from surrounding emitters to remain activated, and the object's presence will not be reported.

The solution to this crossover problem that was used most often in commercially available touch frames before the introduction of the new components was to keep most of the emitter-detector pairs turned off at any given time and to strobe sequentially through each pair, turning on each emitter in sequence to see if the corresponding detector responded. If the detector did not respond, it was assumed that something was blocking the beam, and the number of the beam would be put into a reporting queue. When the touch frame was polled by the computer, it would report in sequence all of the blocked beams. In essence, the infrared LED and the phototransistor in Figure 2 were sequentially replaced, one by one, by every LED and phototransistor in the touch frame. This resulted in several problems. A full scan of the entire screen took between 40 and $100 \mathrm{msec}$. If a large object was blocking many beams, it took longer to scan the entire screen because so many numbers had to be reported and stored. It was impossible to judge whether a large object was blocking the beams or whether a fast-moving object was moving across the screen. In rare cases, a fast-moving response, such as a pigeon peck, could be missed entirely. With the introduction of these new components, another solution suggests itself. Emitters and detectors of different preset pulse frequencies could be used in cases in which the detectors are close enough together to suffer from this crossover problem. Figure 5 shows how this could be arranged with 10 different pulse frequencies used out of a possible 20 . This solution could have been used with the old components, but it would have required at least 10 circuits as complex as the one in Figure 2, plus some additional support circuitry that proved unruly for most commercial products at the time.

\section{Computer Interfacing}

Both the single-beam tapping-box circuit and the multiplebeam touch-frame circuit, as well as many other applications involving these basic infrared components, can be connected to simple, inexpensive, digital I/O boards such as those available from Alpha Products and Metrabyte. All of the circuits presented are TTL-compatible; this means that when the detector senses the emitter's beam, it outputs a nonground voltage, and that when the beam is blocked, it switches the output to ground.

The tapping circuit has been successfully interfaced to a Macintosh SE/30 by connecting the output of the circuit to one pin on an the Alpha Products Digital Input Board (IN-141), which was connected to an Alpha Products serial port controller (SA-129), which was in turn connected to the Macintosh's modem port. The digital input board supplied $5 \mathrm{~V}$ and ground to the circuit and allowed up to eight such circuits to be connected in parallel. The tapping-box circuit was also successfully interfaced to an

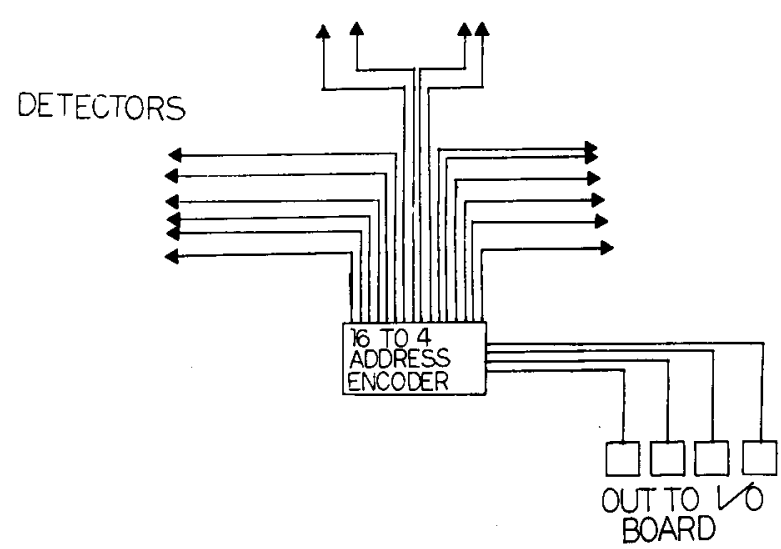

Figure 6. A 16-to- 4 address encoder attached to the detector side of 16 beam-break circuits and to 4 bits of an 8-bit digital 1/O board.

IBM PC/AT computer through the digital I/O ports on a Metrabyte CTM-05 board. The circuit receives $5 \mathrm{~V}$ and ground from the board, and eight circuits can be hooked up in parallel in this case.

In applications in which only one beam will be broken at a time, more than eight circuits can be connected to a single 8-bit $\mathrm{I} / \mathrm{O}$ port by hooking up the outputs of as many as 256 beam-break-detector circuits to an address encoder circuit. An address encoder is a device that polls a number of electrical lines and reports the address of a single line as soon as it is activated. The address of the active line is usually reported as a binary number. In this way, many beams can be set up as a touch frame or as a way to monitor the activity of a subject in a room or a box. Figure 6 shows how this connection can be made from 16 detector circuits to 4 bits of an 8-bit digital I/O board.

\section{Summary}

Through the incorporation of pulse generators and missing-pulse detectors into this set of new optoelectronic components, it has become easier to design circuits resistant to background light, the circuits themselves have become faster, and the need for frequent recalibration has been removed. Because each of these new components generates or responds to one of $\mathbf{2 0}$ different pulse frequencies, the problem of light crossover has been eliminated, and the design of multiple-beam applications has been made much simpler and more reliable.

\section{SELECTED BIBLIOGRAPHY}

Horowitz, P., HILL, W. (1989). The art of electronics. New York: Cambridge University Press.

MIMS, F. M., III (1989). Semiconductor reference guide. Fort Worth, TX: Radio Shack.

MIMS, F. M., III (1986). Engineer's mini-notebook: Opto-electronic Circuits. Fort Worth, TX: Siliconcepts.

Roth, C. H. (1985). Fundamentals of logic design. New York: West. SмIтH, W. J. (1990). Modern optical engineering. New York: McGraw-Hill. 